\title{
Unconjugated Bilirubin Modulates the Intestinal Epithelial Barrier Function in a Human-Derived In Vitro Model
}

\author{
FRANCESCO RAIMONDI, VALERIA CRIVARO, LETIZIA CAPASSO, LUIGI MAIURI, PASQUALE SANTORO, MARIA TUCCI, \\ MARIA VITTORIA BARONE, SERENA PAPPACODA, AND ROBERTO PALUDETTO
}

\author{
Department of Pediatrics [F.R., V.C., L.C., L.M, P.S., M.T., S.P., R.P.], European Laboratory for Food-Induced Diseases [M.V.B.], \\ Università “Federico II," 80131 Naples, Italy
}

\begin{abstract}
Unconjugated bilirubin promotes intestinal secretion without affecting nutrient digestion or absorption. In the current study, the effects of unconjugated bilirubin (UCB) on the barrier function of the intestinal epithelium were investigated. The apical side of human intestinal cell line Caco-2 monolayers was challenged with purified UCB. Transepithelial electrical resistance and paracellular fluxes of $10 \mathrm{kD}$ Cascade blue conjugate dextran were measured. Cell monolayer viability was studied using LDH release and trypan blue exclusion tests. Redistribution of enterocyte tight junction occludin was studied by confocal microscopy. Bilirubin induced a dose-dependent decrease of transepithelial electrical resistance (TEER). This effect was maximal at $6 \mathrm{~h}$ and tended to be reversed at 48 h. Oxidated bilirubin was ineffective. Bilirubin significantly increased fluorescent dextran paracellular passage. Cell viability was not affected by UCB over the 5-200 nmol/L concentration range. Finally, bilirubin triggered a reversible redistribution of tight junctional occludin. UCB increases the permeability of intestinal epithelium. This effect is reversible, dependent on the redox status of the molecule and the rearrangement of the tight junction. These data attribute to bilirubin a novel role of functional modulator of intestinal paracellular permeability in vitro. (Pediatr Res 60: 30-33, 2006)
\end{abstract}

$\mathrm{H}^{5}$ yperbilirubinemia is a common finding in both term and preterm neonates (1). Because bilirubin can cause severe damage to the central nervous system, most investigators have focused their research on its effects on neural cells (2). The interaction of bilirubin with other organs has also been explored, although to a lesser extent. In the intestine, for example, bilirubin is excreted with bile in the gut lumen mainly as mono or diglicuronides. However, the enzyme glycuronidase, of both endogenous and microbial origin, partially converts it to UCB (3). Previous reports have shown UCB concentrations in the intestinal lumen of hyperbilirubinemic newborns to be in the micromolar range $(4,5)$. Investigators have also documented that UCB in the gut does not affect cell viability, nutrient absorption, or brush border enzyme activities, although it can induce watery diarrhea by direct stimulation of ion secretion (6,7). Jaehrig et al. (8) found that UCB transit in the intestinal lumen of the human

Received Setpember 21, 2005; accepted March 12, 2006.

Correspondence: Francesco Raimondi, M.D., Ph.D., Neonatologia, Dipartimento di Pediatria, Università "Federico II" di Napoli, Via S. Pansini 5, 80121 Napoli, Italy; e-mail: raimondi@unina.it

This study was supported by grant 386/2005 Regione Campania.

DOI: 10.1203/01.pdr.0000220344.09034.63 newborn was associated with a decreased transmural potential difference. As the latter is an indirect measurement of the tightness of the intestinal barrier, they suggested that bilirubin could cause "some injury" to the intestinal fence by increasing its permeability. This is of potential clinical interest because a decrease in the gut barrier function has been linked to the pathogenesis of intestinal (i.e. necrotizing enterocolitis) diseases (9) and extraintestinal, particularly allergic and autoimmune, disorders $(10,11)$. The aim of this study was to further investigate in vitro the effect of bilirubin on intestinal epithelial permeability. For this purpose, we have used monolayers of the well-established human transformed cell line Caco-2 that displays many biologic features of the primary intestinal epithelium $(12,13)$. The study was approved by the Institutional Review Board of the "Facoltà di Medicina-Federico II."

\section{MATERIALS AND METHODS}

Reagents. Cell culture chemicals were obtained from GIBCO-Life Technologies (Milan, Italy). $10 \mathrm{kD}$ Cascade blue dextran (14) was purchased from Invitrogen (Carlsbad, CA). UCB was purchased from Sigma Chemical Co. (St. Louis, MO) as mixed isomers and further purified according to McDonagh and Assisi (15). For experimental use, bilirubin was dissolved as a 10 $\mathrm{mmol} / \mathrm{L}$ stock solution in $0.1 \mathrm{~N} \mathrm{NaOH}$. Final concentrations of UCB solutions ranged from 50 to $600 \mathrm{nmol} / \mathrm{L}$ with $\mathrm{pH}=7.4$, after adjusting with chloridric acid. Experiments with bilirubin were performed under a dim light to prevent its photodegradation. Photodegraded UCB (pd-UCB) was prepared in the same fashion but left extensively exposed to full light for at least $12 \mathrm{~h}$. trypan blue and all other reagents of analytical grade were also purchased from Sigma Chemical Co.

Cell culture. Human Caco-2 intestinal cells were purchased from the Istituto Zooprofilattico della Lombardia e dell'Emilia (Brescia, Italy). Cells were grown as previously described $(16,17)$ in Dulbecco modified Eagle medium containing $25 \mathrm{mmol} / \mathrm{L}$ glucose and supplemented with $10 \%$ fetal bovine serum, $1 \%$ nonessential amino acids, $2 \mathrm{mmol} / \mathrm{L}$ L-glutamine, $1 \%$ penicillin-streptomycin, and $1 \%$ sodium pyruvate. Cells were maintained in a humidified atmosphere of $5 \% \mathrm{CO}_{2}$ in air at $37^{\circ} \mathrm{C}$. Single-cell suspensions were obtained from $70-80 \%$ confluent cultures by incubation with $0.05 \%$ trypsin and cells were then seeded at $10^{5}$ cells $/ \mathrm{cm}^{2}$ onto $13-$ or $25-\mathrm{mm}$ glass coverslips, detachable polycarbonate microporous cell culture inserts (Snapwells, 12-mm diameter, 0.4-mm pore size; Costar; Cambridge, MA) and 24-well plates (Multiwell 24 well; Becton \& Dickinson, Franklin Lakes, NJ) according to experimental needs. Since vectorial electrolyte transport requires cells to grow in a polarized fashion with structured intercellular tight junctions, this requires Caco- 2 cells to be cultured for $14 \mathrm{~d}$ before experiments

Abbreviations: pd-UCB, photo-degraded unconjugated bilirubin; TEER, transepithelial electrical resistance; $\mathbf{U C B}$, unconjugated bilirubin 
$(16,17)$. Under experimental conditions, conventional medium was replaced by phenol red- and serum-free medium to avoid additional both protein binding to bilirubin and interferences with fluorescent tracer's measurements. $\mathrm{UCB}$ or experimental medium was added to the apical (luminal) side of the monolayer.

Transepithelial electrical resistance experiments. Measurement of TEER over a 48 -h period was performed as previously described $(16,17)$ by removing the Snapwells from the incubator and allowing a 10-min acclimation under a sterile hood. The entire Snapwell was then placed in a resistance chamber (Snap-Endhom; World Precision Instruments, FL) connected to a voltmeter (Millipore, Billerica, MA). A $1.0-\mathrm{cm}^{2}$ planar electrode was used for all measurements. The screw cap allowed the planar electrode to remain at the same distance from the monolayers in repeated measurements. Monolayers with a basal reading between 350 and $450 \Omega / \mathrm{cm}^{2}$ were used for the study.

Paracellular permeability assay. Measurement of the apical-to-basolateral flux of the paracellular marker $10 \mathrm{kD}$ Cascade Blue dextran through cell monolayers grown on Snapwells was performed as previously described with minor modifications (18). Briefly, after appropriate incubation, UCB solutions and basolateral compartment medium were carefully removed and replaced by phenol red- and serum-free medium containing $1 \mathrm{mg} / \mathrm{mL}$ Cascade Blue dextran in the apical chamber $(0.3 \mathrm{~mL}) ; 1.5 \mathrm{~mL}$ of the same medium without marker was added to the basolateral chamber. After $2 \mathrm{~h}$ of incubation at $37^{\circ} \mathrm{C}$ and $5 \% \mathrm{CO}_{2}$ humidified air, basolateral compartment medium was collected and diluted with phenol red- and serum-free medium. The amount of diffused Cascade Blue dextran was measured with a Perkin Elmer (Wellesley, MA) 2000 fluorescence spectrophotometer (using excitation and emission wavelengths of $365 \mathrm{~nm}$ and $440 \mathrm{~nm}$, respectively).

Cell viability assays. Bilirubin cytotoxicity was investigated using two well-validated assays as the lactate dehydrogenase (LDH) release (19) and the trypan blue exclusion tests (20).

LDH release assay. Caco- 2 cells were cultured on polystyrene, nonpyrogenic, multiwell plates as described above. Control and samples, containing $\mathrm{UCB}$ ranging from 5 to $200 \mathrm{nmol} / \mathrm{L}$, were incubated for 6 and $48 \mathrm{~h}$ at $37^{\circ} \mathrm{C}$, $95 \%$ relative humidity, $5 \% \mathrm{CO}_{2}$. Tissue culture media were then collected and centrifuged at 14,000 rpm for $60 \mathrm{~s}$ and supernatants stored no more than $12 \mathrm{~h}$ at $4{ }^{\circ} \mathrm{C}$ before the assay. In each plate, a single well received a $2 \%$ Triton X-100 solution, which represents the maximum LDH release from the monolayer. A colorimetric, endpoint assay obtained from Roche Diagnostic GmbH (Penzberg, Germany) was used to quantitate the amount of enzyme in each sample. The amount of the end-product formazane produced was read as optical density (OD) by a spectrophotometer at $\lambda=500 \mathrm{~nm}$. Because UCB shows a slight absorption at $\lambda=492 \mathrm{~nm}$, from each sample the corresponding OD of the UCB was subtracted. Results are expressed as a percentage of the maximum LDH release.

Trypan blue exclusion dye test. Trypan blue exclusion dye test was also used to determine UCB toxicity on Caco-2 cells grown in multiwell plates. Control and samples, containing UCB ranging from 5 to $600 \mathrm{nmol} / \mathrm{L}$, were incubated 6 and $48 \mathrm{~h}$ at $37^{\circ} \mathrm{C}, 95 \%$ relative humidity, $5 \% \mathrm{CO}_{2}$. A $2 \%$ trypan blue solution in assay medium was added to the monolayer for $10 \mathrm{~min}$ and the stained cells were counted from 12 different fields at a magnification of $\times 200$.

Tight junction occludin microscopy. Caco- 2 cells were grown on glass coverslips, challenged with $50 \mathrm{nmol} / \mathrm{L}$ bilirubin for 6 or $48 \mathrm{~h}$ and then rinsed three times with $0.01 \mathrm{~mol} / \mathrm{L} \mathrm{pH} 7.4 \mathrm{PBS}$. Cells were fixed in $3 \%$ paraformaldehyde for $15 \mathrm{~min}$, permeabilized with $0.2 \%$ Triton X-100 for $5 \mathrm{~min}$ and then rinsed again in PBS. Staining with monoclonal fluorescent fluorescein isothiocyanate anti-human occludin antibody (Invitrogen) 1:50 was performed overnight. Then monolayers were washed three times got $10 \mathrm{~min}$ in 0.01 $\mathrm{mol} / \mathrm{L}$, pH 7.4, of PBS, mounted, and then examined using a confocal fluorescence microscope (Zeiss, LSM 510, Leipzig, Germany).

Statistical analysis. Results are presented as mean \pm SD of a minimum of five experiments. The data were analyzed with the help of a commercial software (GraphPad PRISM; GraphPad Software Incorporated, San Diego, CA). All data sets passed the normality test and the one-way analysis of variance, together with the Tukey post hoc test was used to compare different groups of data. A $p<0.05$ was considered statistically significant. Similar results were obtained analyzing the data by nonparametric tests (KruskalWallis together with the Dunn posttest).

\section{RESULTS}

TEER experiments. A decrease in TEER is indirect but generally accepted evidence that the permeability between adjacent enterocytes (i.e. paracellular) has increased $(16,17,21)$. UCB induced a dose-dependent decrease of TEER in the $0-200 \mathrm{nmol} / \mathrm{L}$ concentration range and reaching a plateau soon thereafter (Fig. 1A). The decrease of TEER was maximal after $6 \mathrm{~h}$ and tended to be reversible over $24 \mathrm{~h}$. pd-UCB $(200 \mathrm{nmol} / \mathrm{L})$ was not effective in modulating TEER.

Paracellular permeability assay. To add a direct evidence of the effect of UCB on the paracellular pathway, the flux of $10 \mathrm{kD}$ cascade blue dextran was investigated. After a 6-h incubation, UCB promoted the apical-to-basolateral flux of Cascade Blue dextran starting at a concentration of $50 \mathrm{nmol} / \mathrm{L}$. Paracellular movement of Cascade Blue dextran across Caco-2 cells monolayers progressively increased up to the concentration of $150 \mathrm{nmol} / \mathrm{L}$ (Fig. $2 A$ ). Dextran flux after $48 \mathrm{~h}$ was comparable to baseline flux, indicating that UCB-induced permeability increase is reversible (Fig. $2 B$ ). Finally, 50 $\mathrm{nmol} / \mathrm{L}$ UCB time-dependent dextran accumulation is shown in Figure $2 C$.

Cell viability assay. To exclude that the increased permeability was not due to a monolayer disruption, Caco-2 cells were incubated for 6 or $48 \mathrm{~h}$ with UCB. Regardless of the incubation time, UCB concentrations up to $200 \mathrm{nmol} / \mathrm{L}$ did not affect cell viability as assessed by both LDH release (Fig. 3A) and trypan blue (Fig. $3 B$ ) tests. A UCB concentration of 600 $\mathrm{nmol} / \mathrm{L}$ was significantly cytotoxic by both assays; 600 $\mathrm{nmol} / \mathrm{L}$ pd-UCB was not cytotoxic by both assays.

Tight junction occluding microscopy. Confocal microscopy allows scanning of thin planes of a sample along the $Z$ optical axis by controlled micrometer increments. Results of a typical study of treatment of Caco-2 cells with UCB are shown in Figure $4(n=7)$. In Figure $4 A$, the scanning from top to bottom of an untreated Caco-2 monolayer shows a distinct, continuous line of fluorescence spanning through a limited number of sections (sections $4,5,6$ ), describing a homogeneous occludin ring along the entire perimeter of the cells. Figure $4 B$ shows a monolayer treated for $6 \mathrm{~h}$ with 50 $\mathrm{nmol} / \mathrm{L}$ UCB. Most of the planes show a patchy organization of intracellular occludin, and its staining is not limited to some sections, but it can be observed in almost all the optical sections, suggesting that a significant occludin redistribution had occurred. In a separate set of experiments $(n=5)$, the reversibility of occludin redistribution was investigated: after $48 \mathrm{~h}$ of treatment with $50 \mathrm{nmol} / \mathrm{L} \mathrm{UCB}$; the organization of occludin showed a regular pattern comparable to the not treated sample (not shown). The timing of these microscopy observations closely correlates with normalization of TEER
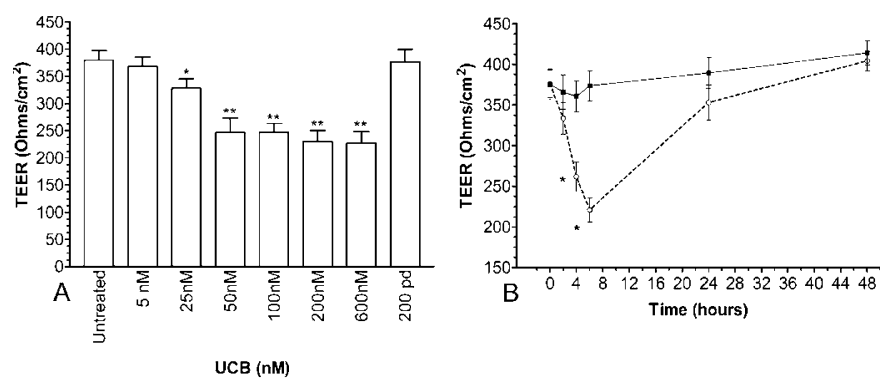

Figure 1. Luminally applied UCB $(6 \mathrm{~h})$ decreases Caco-2 monolayers TEER in a dose-dependent fashion $(A) ; 200 \mathrm{nM}$ photo-degraded UCB (200pd) is not effective. $(B)$ Time course of $50 \mathrm{nmol} / \mathrm{L}$ luminally applied UCB. The effect develops over the first $6 \mathrm{~h}$ and shows a trend to reversibility within 48 hours. $n=15 ; * p<0.05 v s$ vehicle-treated monolayers; ** $p<0.05$ vs UCB $50 \mathrm{nM}$. 

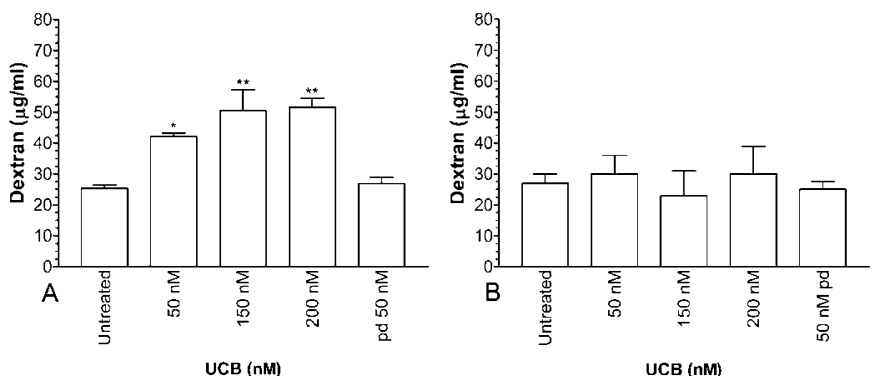

Figure 2. UCB promotes a dose-dependent paracellular passage of $10 \mathrm{kDa}$ cascade blue ${ }^{\circledR}$ Dextran. As little as $50 \mathrm{nmol} / \mathrm{L}$ UCB allows the recovery at the monolayer's basolateral side of a significant dextran concentration after a $6 \mathrm{~h}$ incubation period. (A). UCB-treated cells for $48 \mathrm{~h}$ show no increased paracellular marker flux compared to untreated monolayers $(B) n=10 ;{ }^{*} p<0.05$ vs untreated cells; **p $<0.05$ vs UCB $50 \mathrm{nM}$.

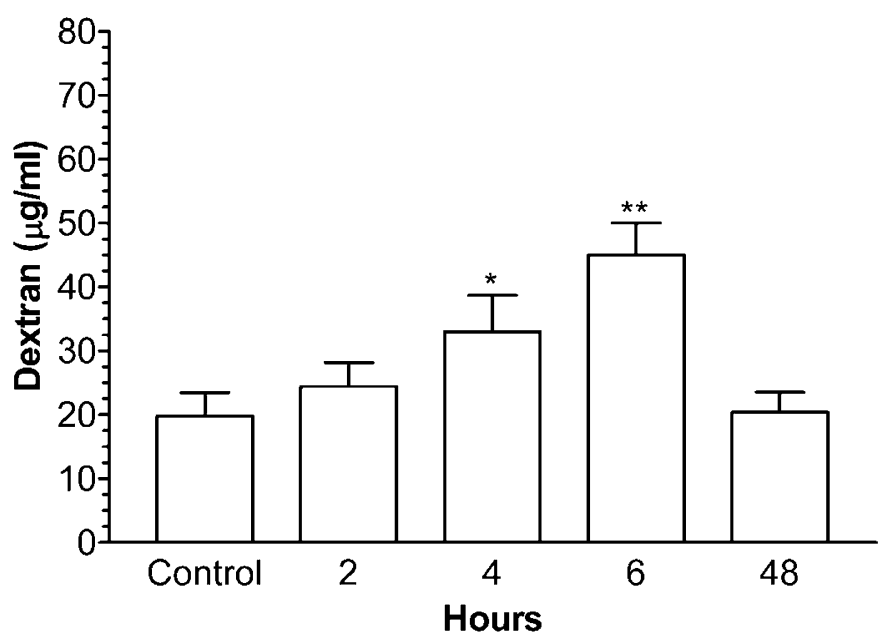

Figure 3. Time-dependent dextran accumulation in $50 \mathrm{nmol} / \mathrm{L} \mathrm{UCB}$ treated monolayers: a significant increment of dextran concentration measured at the monolayer's basolateral side is observed after 4 h. $n=10$; ${ }^{*} p<0.05 v s$ untreated cells; ** $p<0.05$ vs UCB $50 \mathrm{nmol} / \mathrm{L} 4 \mathrm{~h}$ incubation.

and paracellular flux data, pointing to occludin as a major player in the UCB modulation of paracellular permeability.

\section{DISCUSSION}

The present article characterizes the in vitro effect of UCB on differentiated Caco-2 monolayers. While most investigators have previously studied bilirubin interplay with the gut using a commercial UCB preparation, (22), we further purified commercial UCB to eliminate the nonphysiologic isomers (15). In biologic systems, UCB mostly binds albumin and other proteins, although it is the unbound or "free" UCB that is now thought to be biologically active (23). Our experiments were performed under serum-free conditions to pinpoint the role of free, purified UCB in modulating the intestinal epithelial fence function. Ostrow et al. (24) have recently reviewed the dose-dependent aggregation of free UCB. The latter molecule has an aqueous solubility limit around $70-100 \mathrm{nmol} / \mathrm{L}$ above which it forms oligomers that are too small to precipitate but that can reversibly dissociate and serve as reservoirs to replenish UCB monomers removed by the cells. Concentrations above $500 \mathrm{nmol} / \mathrm{L}-1 \mu \mathrm{mol} / \mathrm{L}$ give raise to larger aggregates that are generally associated with cellular toxicity
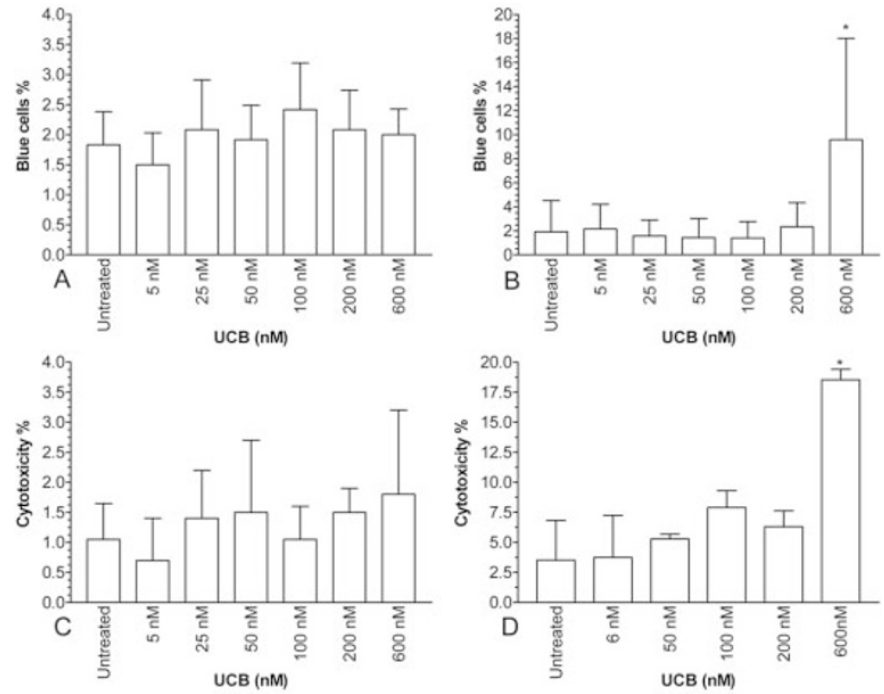

Figure 4. Caco-2 cells monolayers were exposed for $6 \mathrm{~h}$ or $24 \mathrm{~h}$ to increasing concentrations of UCB and cytotoxicity was investigated by Trypan blue ( $A$ and $B$ ) exclusion and the LDH release $(C$ and $D)$ tests. Bilirubin concentrations that decrease TEER do not affect cell viability in the $50-200 \mathrm{nmol} / \mathrm{L}$ range; $600 \mathrm{nmol} / \mathrm{L}$ UCB displays significant cytotoxicity regardless of the incubation time. $n=5 ; * p<0.05$.

(25). Remarkably, we found that the dose-response effect on permeability assessed by TEER monitoring and dextran flux experiments was present not far from the aqueous solubility range of free UCB. The plateau at $100-150 \mathrm{nmol} / \mathrm{L}$ (Figs. $1 A$ and 2) may correspond to the formation of the cited small-size aggregates. Larger concentrations showed incipient cytotoxicity (Fig. 3). There is then a UCB concentration range where the increased permeability is obtained in an intact monolayer. These data are also in agreement with previous work on the intestinal effects of UCB. Using an in vivo perfusion animal model, Whitington et al. (6) demonstrated that bilirubin induced an active secretion of water and electrolytes without damaging the mucosa. A similar conclusion is suggested by Guandalini et al. (7), who described UCB induced fluid secretion in presence of normal nutrient absorption. In all cases, then, UCB intestinal effects were not dependent on its cytotoxicity.

Using dextran as a not absorbable marker, we show that UCB modulates the Caco- 2 cells monolayer paracellular permeability. Timing of dextran fluxes and TEER experiments closely correlate with our confocal microscopy observations, strongly suggesting that occludin, a transmembrane component of the tight junction, may be the terminal agent of the UCB-induced increased paracellular permeability. An occludin-driven modulation of gut permeability has already been demonstrated for molecules of diverse origin and chemical nature (26-28). Some of these modulators belong to redox active species (29). Since pd-UCB was not effective (Fig. 1A), the redox status also of this molecule seems to be important. UCB oxidation might explain the reversible nature of the permeability increase. As a complementary hypothesis, part of UCB molecules might be pumped out of the cell by carriers that are expressed in this cell line (30).

As nanomolar UCB concentrations are normally present in the gut in vivo $(5,6)$, we believe that the UCB modulation of 

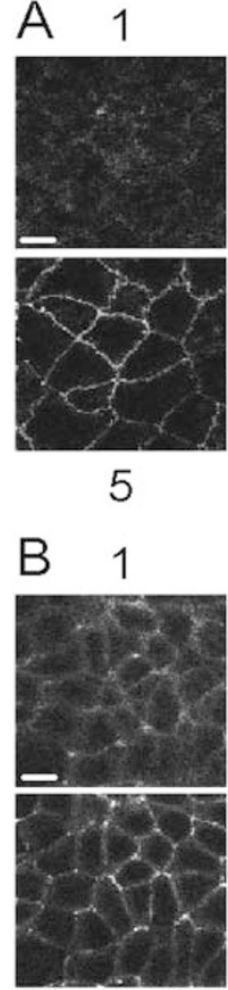

5

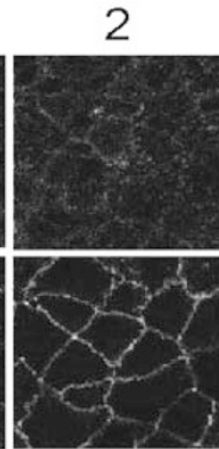

6

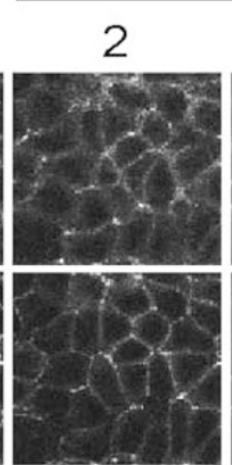

6

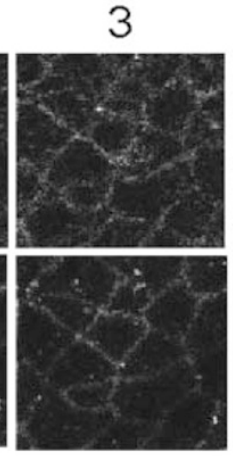

7

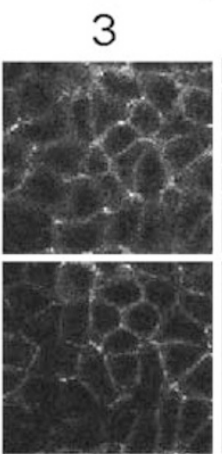

7

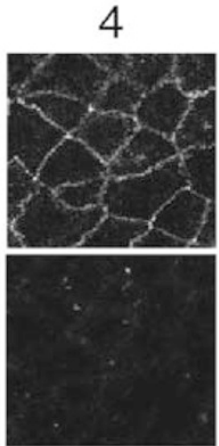

8

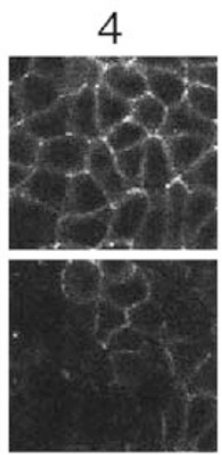

8
Figure 5. UCB induces a marked redistribution of intracellular occludin. Optical sections along the $Z$ axis $(1 \mu \mathrm{m})$. (A) Untreated Caco-2 cells show a linear distribution of occludin along the cell perimeter restricted to sections 4 , 5 , and 6. (B) In monolayers incubated for $6 \mathrm{~h}$ with $50 \mathrm{nmol} / \mathrm{L} \mathrm{UCB}$, no organized structure was detected and fluorescence was distributed in a scattered and clusterized fashion along all sections $(40 \times$ objective $)$. Scale bar $=$ $20 \mu \mathrm{m}$.

intestinal permeability is of possible physiologic relevance We further speculate that when the UCB-induced permeability increase becomes sustained, as in unconjugated hyperbilirubinemia, a prolonged transepithelial passage of small bacterial products and food allergens may occur with possible clinical implications. In this respect, a link between intestinal permeability modulators and human disease is now emerging from several lines of evidence. Tumor necrosis factor- $\alpha$, for instance, is responsible for the gut barrier dysfunction in Crohn's disease (31), while other inflammatory molecules sustain the increased intestinal permeability in patients suffering from food allergy $(27,32)$.

In conclusion, we have shown that nanomolar concentrations of protein-unbound, undegraded UCB regulate the space between adjacent enterocytes. UCB can be then considered, at least in vitro, a functional modulator of gut paracellular permeability.

\section{REFERENCES}

1. American Academy of Pediatrics Subcommittee on Hyperbilirubinemia 2004 Management of hyperbilirubinemia in the newborn infant 35 or more weeks of gestation. Pediatrics 114: 297-316

2. Ostrow JD, Pascolo L, Brites D, Tiribelli C 2004 Molecular basis of bilirubininduced neurotoxicity. Trends Mol Med 10:65-70

3. Dennery PA, Seidman DS, Stevenson DK 2001 Neonatal hyperbilirubinemia. N Engl J Med 344:581-590
4. Keshavan P, Schwemberger SJ, Smith DL, Babcock GF, Zucker S 2004 Unconjugated bilirubin induces apoptosis in colon cancer cells by triggering mitochondrial depolarization. Int J Cancer 112:433-445

5. Kotal P, Van der Veere CN, Sinaasappel M, Elferink RO, Vitek L, Brodanova M, Jansen PL, Fevery J 1997 Intestinal excretion of unconjugated bilirubin in man and rats with inherited unconjugated hyperbilirubinemia. Pediatr Res 42:195-200

6. Whitington PF, Olsen WA, Odell GB 1981 The effect of bilirubin on the function of hamster small intestine. Pediatr Res 15:1009-1014

7. Guandalini S, Fasano A, Albini F, Marchesano G, Nocerino A, De Curtis M 1988 Unconjugated bilirubin and the bile from light exposed Gunn rats inhibit intestinal water and electrolyte absorption. Gut 29:366-371

8. Jaehrig K, Ballke EH, Koenig A, Meisel P 1987 Transepithelial electric potential difference in newborns undergoing phototherapy. Pediatr Res 21:283-284

9. Kelly N, Friend K, Boyle P, Zhang XR, Wong C, Hackam DJ, Zamora R, Ford HR, Upperman JS 2004 The role of glutathione antioxidant system in gut barrier failure in a rodent model of experimental necrotizing enterocolitis. Surgery 136:557-566

10. Farhadi A, Banan A, Fields J, Keshavarzian A 2003 Intestinal barrier: an interface between health and disease. J Gastroenterol Hepatol 18:479-497

11. DeMeo MT, Mutlu EA, Keshavarzian A, Tobin MC 2002 Intestinal permeation and gastrointestinal disease. J Clin Gastroenterol 34:385-396

12. Grasset E, Pinto M, Dussaulx E, Zweibaum A, Desjeux JF 1984 Epithelial properties of human colonic carcinoma cell line Caco-2: electrical parameters. Am J Physiol 247:C260-C267

13. Pinto M, Robin-Leon S, Appay MD, Kedinger M, Triadou N, Dussaulx E, Lacroix B, Simon-Assmann P, Haffen K, Fogh J, Zweibaum A 1983 Enterocyte-like differentiation and polarization of the human colon carcinoma cell line Caco-2 in culture. Biol Cell (Paris) 47:323-330

14. Whitaker JE, Haugland RP, Moore PL, Reese M, Haugland RP 1991 Cascade blue derivative: water soluble, reactive, blue emission dye evaluated as fluorescent labels and tracers. Anal Biochem 198:119-130

15. McDonagh AF, Assisi F 1971 Commercial bilirubin: a trinity of isomers. FEBS Lett 18:315-317

16. Raimondi F, Kao JP, Fiorentini C, Fabbri A, Donelli G, Gasparini N 2000 Enterotoxicity and cytotoxicity of Vibrio parahaemolyticus thermostable direct hemolysin in in vitro systems. Infect Immun 68:3180-3185

17. Raimondi F, Santoro P, Maiuri L, Londei M, Annunziata S, Ciccimarra F 2002 Reactive nitrogen species modulate the effects of rhein, an active component of senna laxatives, on human epithelium in vitro. J Pediatr Gastroenterol Nutr 34:529 534

18. Sonoda N, Furuse M, Sasaki H, Yonemura S, Katahira J, Horjuchi Y 1999 Clostridium perfringens enterotoxin fragment removes specific claudins from tight junction strands: evidence for direct involvement of claudins in tight junction barrier. J Cell Biol 147:195-204

19. Fernandes A, Silva RF, Falcao AS, Brito MA, Brites D 2004 Cytokine production, glutamate release and cell death in rat cultured astrocytes treated with unconjugated bilirubin and LPS. J Neuroimmunol 153:64-75

20. Banan A, Smith GS, Deshpande Y, Rieckenberg CL, Kokoska ER, Miller TA 1999 Prostaglandins protect human intestinal cells against ethanol injury by stabilizing microtubules: role of protein kinase $\mathrm{C}$ and enhanced calcium efflux. Dig Dis Sci 44:697-707

21. Weisiger RA, Ostrow JD, Koehler RK, Webster CC, Pascolo L, Tiribelli C 2001 Affinity of human serum albumin for bilirubin varies with albumin concentration and buffer composition: results of a novel ultrafiltration method. J Biol Chem 276:29953-29960

22. Li BU, Whitington PF, Odell GB 1984 The reversal of bilirubin-induced intestinal secretion by agar. Pediatr Res 18:79-82

23. Vitek L, Kotal P, Jirsa M, Malina J, Cerna M, Chmelar D, Fevery J 2000 Intestinal colonization leading to fecal urobilinoid excretion may play a role in pathogenesis of neonatal jaundice. J Pediatr Gastroenterol Nutr 30:294-298

24. Ostrow JD, Mukerjee P, Tiribelli C 1994 Structure and binding of unconjugated bilirubin: relevance for physiological and pathophysiological function. J Lipid Res 35:1715-1737

25. Ostrow JD, Pascolo L, Tiribelli C 2003 Reassessment of the unbound concentrations of unconjugated bilirubin in relation to neurotoxicity in vitro. Pediatr Res 54:98-104

26. Fasano A, Fiorentini C, Donelli G, Uzzau S, Kaper JB, Margaretten K, Ding X, Guandalini S, Comstock L, Goldblum SE 1995 Zonula occludens toxin modulates tight junctions through protein kinase $\mathrm{C}$-dependent actin reorganization, in vitro. J Clin Invest 96:710-720

27. Heyman M, Desjeux JF 2000 Cytokine-induced alteration of the epithelial barrier to food antigens in disease. Ann N Y Acad Sci 915:304-311

28. Banan A, Fields JZ, Zhang Y, Keshavarzian A 2001 iNOS upregulation of F-actin and barrier of intestinal monolayers. Am J Physiol Gastrointest Liver Physiol 280:G1234-G1246

29. Vallette G, Jarry A, Bronza JE, Laboisse CL 1997 iNO-dependent and iNOindependent IL-1 production by a human colonic epithelial cell line under inflammatory stress. Br J Pharmacol 121:187-192

30. Tiribelli C, Ostrow JD 2005 The molecular basis of bilirubin encephalopathy and toxicity: report of an EASL Single Topic Conference, Trieste, Italy, 1-2 October, 2004. J Hepatol 43:156-166

31. Söderholm JD, Olaison G, Peterson KH, Franzén LE, Lindemark T, Wirém L, Tagesson C, Sjo"dahl R 2002 Augmented increase in tight junction permeability by luminal stimuli in the non-inflamed ileum of Crohn's disease. Gut 50:307-313

32. Konishi Y 2003 Modulations of food-derived substances on intestinal permeability in Caco-2 cells monolayer. Biosci Biotechnol Biochem 67:2297-2299 